\title{
Summary of ECRH/ECCD Presentations at EC18
}

\author{
Kazunobu Nagasaki ${ }^{1, a}$ \\ ${ }^{1}$ Institute of Advanced Energy, Kyoto University, Gokasho, Uji, Kyoto 611-0011, Japan
}

\begin{abstract}
A summary of ECRH/ECCD session in EC18 is made. 28 contribution papers (15 oral and 13 presentations) are presented, which include an overview, a variety of ECRH/ECCD applications, systems and wave physics.
\end{abstract}

\section{Overview}

Goodman gave an invited talk on recent physics studies using ECRH/ECCD at the 2nd and 3rd harmonics in the TCV tokamak. TCV has 7 independently controlled launching antennas and $9 \mathrm{~mm}$-wave sources $(6$ at $82.7 \mathrm{GHz}$ and 3 at $118 \mathrm{GHz}$, each at $0.5 \mathrm{MW}$ and $2 \mathrm{~s}$ pulse duration). A variety experiments using this ECRH/ECCD system were presented: sawtooth control techniques for NTM pre-emption, time-resolved rotation measurements during a sawtooth cycle, non-stiffness of edge transport in L-mode, robust control of NTMs with real-time equilibria (RT-LIUQE), ELM control example related to the rotation measurements, EC power transmission measurements. An interesting ECRH application is that EC-locked sawteeth allowed measurement of the co-current direction core rotation kick at the ST crash. Future EC system upgrade planned for the next 4 years was also presented.

\section{ECRH/ECCD applications}

\subsection{Confinement and transport}

In ITER, dominant electron heating by alpha particles increases $T_{e} / T_{i}$, affecting transport properties. Stober reported that substituting ion heating by electron heating increased energy transport in the plasma core in moderate collisionalities in AUG. Almost $4 \mathrm{MW}$ of ECRH power in the plasma were available, delivered from 7 gyrotrons at $140 \mathrm{GHz}$. The results demonstrate the crucial role of $T_{e} / T_{i}$ for the ITG dominated transport over a wide range of $T_{e} / T_{i}$. Modeling with TGLF reproduced profiles and time evolution quite well. Central electron heating of ITG-dominated plasmas increases transport in the ionchannel due to the inevitable increase of $T_{e} / T_{i}$.

The effect of ECRH on transport was investigated also in the DIII-D tokamak. Pinsker gave a talk on application of ECRH to the study of transport in ITER baseline scenario-like discharges. Adding ECRH power increased fluctuation levels in the core region, inducing higher heat, particle and momentum diffusivities. Modeling shows that chief culprit appears to be reduction in $\mathrm{E} \times \mathrm{B}$ shearing from reduction in rotation. The result is consistent with 'standard model' of turbulent transport. Measured fluctuations appear to qualitatively support the turbulent transport model. In some cases, density scaling of confinement with EC appears to be stronger than $\mathrm{H}_{98}(\mathrm{y}, 2)$ scaling. Puffing to compensate for the pump-out can improve the confinement significantly

Mishra gave a talk on high $\beta_{p}$ plasma formation using off-axis ECCD in Ohmic heated plasma in the QUEST spherical torus (ST). High poloidal beta plasma $\left(\varepsilon \beta_{p} \sim\right.$ 1.6) was produced and sustained by ECRH/ECCD fully non-inductively and in $\mathrm{OH}$ heated plasma. With high $\beta_{p}$, plasma configuration was naturally transformed to inboard poloidal null (IPN) configuration from inboard limiter configuration. Intrinsic plasma rotation along toroidal direction was observed in high $\beta_{p}$ IPN plasma in non-inductive scenario without any external momentum input.

\subsection{Plasma start-up}

Reliable plasma start-up in superconducting tokamaks is an important issue due to low toroidal loop voltage. Plasma start-up using second harmonic X-mode (X2) is also a topic in helical devices. A special session was made for start-up using ECRH with 5 oral talks.

Preynas compared experimental results on plasmastart-up using X2 ECRH in Heliotron J, LHD and WEGA, and predicted necessary conditions for plasma start-up in W7-X. Experimental results from the 3 devices are in good agreement. High confidence is given in X2 ECRH plasma start-up as reliable and robust method for the 1st operation phase of W7-X.

Tanaka reported on experimental results on plasma start-up without use of center solenoid in KSTAR. The

\footnotetext{
${ }^{\mathrm{a}}$ Corresponding author: nagasaki.kazunobu.4x@kyoto-u.ac.jp
} 
plasma current was started and ramped up to $14.5 \mathrm{kA}$ without use of center solenoid by injecting $84 \mathrm{GHz}$ oblique fundamental O-mode of $180 \mathrm{~kW}$ power. The cross-field-passing-electron (CFPE) model shows that CFPE current is driven with addition to the pressure driven current and produces the initial closed flux surfaces.

Uchida presented a talk on formation of extremely overdense plasmas by electron Bernstein waves (EBW) in the LATE ST. $\mathrm{I}_{\mathrm{p}}$ reached up to $12 \mathrm{kA}$ at the electron density more than 7 times higher than cutoff, by 2.45 $\mathrm{GHz} 60 \mathrm{~kW}$ power. Heating of bulk electrons as well as the current carrying fast electrons $(\sim 100 \mathrm{keV})$ was realized by EBWs. Large magnetic fluctuation events were observed at $I_{\mathrm{p}}>10 \mathrm{kA}$, accompanying ejection of plasma across the LCFS. Avoidance or mitigation of these events is required to reach higher $\mathrm{I}_{\mathrm{p}}$.

Shevchenko gave a talk on long pulse EBW start-up experiments on MAST. They achieved plasma current of $73 \mathrm{kA}$ which was more than twice higher than the previous experimental result of $33 \mathrm{kA}$. Closed flux surfaces (CFS) were observed on fast video indicating that tokamak-like equilibrium was established during the EBW start-up phase. Anti-phase response to vertical modulation suggests that current was driven by EBWs. Power scan indicates that generated plasma current after CFS formation depends linearly on RF power injected.

Shukla reported on $42 \mathrm{GHz}$ ECRH assisted plasma breakdown in the SST-1 tokamak. O1 or X2 was launched from the low field side, and successful breakdown was achieved in both cases. Delay around $40 \mathrm{~ms}$ was observed in case of X2 assisted breakdown. The breakdown was achieved at low loop voltage $\sim 2.8 \mathrm{~V}$ and the corresponding electric field is comparable with ITER requirement.

\subsection{MHD control}

Recently ECRH/ECCD is applied for stabilizing energetic-particle-driven MHD modes as well as sawteeth and tearing modes. Nagasaki presented experimental results in Heliotron $J$ and LHD that ECRH/ECCD is an effective scheme to control energetic-particle-driven MHD modes. Both co-ECCD and counter-ECCD were effective at stabilizing energetic particle modes (EPMs). Alfven Eigenmodes (AEs) were stabilized/destabilized by ECRH in LHD, depending on the magnetic field conditions and EC power. ECRH/ECCD appears to affect the driving and damping terms of EP-driven modes.

Stober presented feedback-controlled stabilization of NTMs on AUG using fast movable ECRH launchers. Real-time correlation analysis of $1 \mathrm{MHz}$ ECE and Mirnov data allows to determine the mode position in real-time. The O-point heating with $50 \%$ duty cycle was more efficient than $\mathrm{CW}$-heating, which was more efficient than X-point heating with $50 \%$ duty cycle. Multiple NTM modes were stabilized in a single plasma shot by controlling the launcher mirror.

Joung presented development of real-time NTM control system with ECRH/ECCD in KSTAR. Active feedback control of ECRH includes feedback control of the deposition position of ECRH power by the fast mirror movement, real-time ray-tracing calculation of EC beam, and real-time detection of the EC deposition location. The first NTM suppression experiment was performed with the PLC launcher controller. The observed NTM was $\mathrm{n}=1$ mode with $\mathrm{f} \sim 10 \mathrm{kHz}$ at $\mathrm{Bt}=2.6 \mathrm{~T}, \mathrm{I}_{\mathrm{p}}=600 \mathrm{kA}, \kappa$ $\sim 1.6$. They tried to suppress the NTM by feed-forward antenna control, and demonstrated its partial suppression.

In the presentation by Jeong, control of sawtooth period with EC waves was demonstrated in KSTAR plasmas. The sawtooth was destabilized by on-axis coECCD, while stabilization of the sawtooth was observed by off-axis co-ECCD at the outside of $\mathrm{q}=1$ surface. Sawtooth period can be controlled in accurate way by EC beam injection conditions.

\subsection{Steady-state operation}

In a talk by Idei, non-inductive current drive experiments using $28 \mathrm{GHz}$ ECRH in the QUEST spherical torus were presented. ECCD effect was observed in Ohmically heated plasmas with feedback regulation of Center Solenoid (CS) coil current in X2 inboard off-axis heating scenario. In non-inductive current drive experiments only by the $28 \mathrm{GHz}$ injection, $54 \mathrm{kA}$ plasma current was sustained for $0.9 \mathrm{~s}$ ECRH. High non-inductive plasma current of $66 \mathrm{kA}$ was also attained by the $28 \mathrm{GHz}$ ECRH/ECCD. Density jump across $8.2 \mathrm{GHz}$ cutoff density was observed in superposed $28 \mathrm{GHz} / 8.2 \mathrm{GHz}$ injections. The $50 \mathrm{kA}$ plasmas were sustained by the 8.2 $\mathrm{GHz}$ injection to the $28 \mathrm{GHz}$ target plasma.

Yoshimura showed recent progress on steady-state operation in LHD. Using two $77 \mathrm{GHz}$ gyrotrons alternately at intervals of two minutes and an $84 \mathrm{GHz}$ gyrotron continuously, a $30 \mathrm{~min}$. Long-pulse discharge with $\mathrm{n}_{\mathrm{e}}=0.7 \times 10^{19} \mathrm{~m}^{-3}$ and $\mathrm{T}_{\mathrm{e}}(0)=1.5 \mathrm{keV}$ was achieved by the time-averaged injection power of $240 \mathrm{~kW}$ in the 2012 campaign. In the 2013 campaign using 3 gyrotrons of $154 \mathrm{GHz}$, the improved $77 \mathrm{GHz}$ and $84 \mathrm{GHz}$ with 380 $\mathrm{kW}$ in total, higher temperature plasma with internal transport barrier was sustained for 12 min with $\mathrm{n}_{\mathrm{e}}=$ $0.8 \times 10^{19} \mathrm{~m}^{-3}$ and $\mathrm{T}_{\mathrm{e}}(0)=4 \mathrm{keV}$.

\section{Systems}

In Lohr's paper, presented by Pinsker, performance history and upgrades for the DIII-D gyrotron complex were reported. The DIII-D $110 \mathrm{GHz}$ ECH system is running 5 gyrotrons with a 6th being conditioned. Injected EC power decreased from 3.5 MW in 2013 to 2.8 MW. Two gyrotrons failed during 2013, and a new gyrotron "Vader" at $117.5 \mathrm{GHz}$ had good EC performance in CPI tests with 1.8 MW short pulses, 1.5 MW short pulses, and good mode stability for new TE 20,9 mode. This new frequency gyrotron will give better ECCD performance at highest DIII-D field. High speed mirror motion capability was installed and operating. Power monitor/mode analysis was tested and being installed at miter bends. NASA microwave rocket tests was performed in a closed environment. A new $110 \mathrm{GHz}$ CPD gyrotron is being conditioned. 
Schubert gave a talk on machine safety issues related to stray radiation. Damages of in-vessel components by non-absorbed microwaves on AUG were reported during regular inspections. Absorbing insulating materials which absorb microwaves were degraded, and local damages, even of metallic components, were observed on surfaces which were exposed directly to the microwave beam. The ways how to enhance machine safety are to pay attention to primary beam path and non-absorbed microwave fraction, hardening of inner heat shield, control and monitoring of wave polarization, and stray radiation level monitoring in the vicinity of the launchers and near possible beam trajectories.

Igami gave a talk on recent upgrading of ECRH system and studies to improve ECRH performance in the LHD. $4 \mathrm{MW}$ injection (4.5 MW max.) was available for short pulse experiments. The injection of $0.3 \mathrm{MW} / 270 \mathrm{~s}$ was achieved at a horizontal antenna in long pulse experiment using a $77 \mathrm{GHz} \mathrm{CW}$ gyrotron. The experimental result suggests that $\mathrm{O}-\mathrm{X}$ mode coupling caused by magnetic shear in the edge region including the ergodic region should be taken into account for high single-pass absorption.

Nishiura gave a talk on application of ECRH to the ring trap device, RT-1 The X-mode injection of $8.2 \mathrm{GHz}$, $50 \mathrm{~kW}$ enhanced the heating efficiency compared with the O-mode injection. The density at the fundamental resonance may exceed the cut-off density. In the high beta operation with electron density higher than $4 \times 10^{17}$ $\mathrm{m}^{-3}$, magnetic fluctuations with a few $10 \mathrm{kHz}$ were observed.

Wang reported on power measurement system of ECRH on HL-2A. Two methods are used or being developed for measuring the power of ECRH system. One is a calorimetric technique, which is running successfully in commissioning and ECRH experiment. The other is use of a directional coupler, which is now under development.

Seok presented real-time feed-backed anode power system for $170 \mathrm{GHz}$ gyrotron in KSTAR. The new anode power supply has cap charge power supply (CCPS) and high voltage switch (HVS). The output of CCPS is rapidly feedback-controlled by real time control. The APS modulation is possible to up to $10 \mathrm{kHz}$ with the stability of $\pm 0.3 \%$. The new anode power supply was found to be suitable for gyrotron application.

Platania presented beam propagation and stray radiation in ITER ECRH/ECCD upper launcher. In order to study beam propagation, detrimental effects, straylight behavior and to verify analytical calculations, a 3-D model of the UL optical system was implemented with the electromagnetic code GRASP ${ }^{\circledR}$. The distortions due to crosstalk were evaluated. The main directions of propagation from and around the steering mirrors were identified and the impact on surrounding structures was quantified.

Purohit presented instrumentation and control system architecture for ITER. The ECRH/ECCD system consists of 24 gyrotrons, 12 sets of high voltage power supplies, and 24 transmission lines which connect the gyrotron to either of 4 upper launchers or 1 equatorial launcher. Highlighted were functional study, architecture design of
EC control system, CODAC standards, and challenges to ECCS and mitigation strategy.

Taylor presented a plan of a MW-level $28 \mathrm{GHz}$ ECRH for NSTX-U. Simulations of $28 \mathrm{GHz}$ ECRH of a Coaxial Helicity Injection (CHI) start-up plasma predict rapid core heating and reduced plasma current $\left(\mathrm{I}_{\mathrm{p}}\right)$ decay. A CHI was successfully used for non-inductive plasma start-up in NSTX, but $\mathrm{T}_{\mathrm{e}}(0)$ was very low $(\sim 10 \mathrm{eV})$ resulting in rapid $\mathrm{I}_{\mathrm{p}}$ decay. A 1-2 MW, $28 \mathrm{GHz}$ ECRH system is planned for NSTX-U in 2017-18 and it will initially be used to increase $\mathrm{T}_{\mathrm{e}}(0)$ during $\mathrm{CHI}$ start-up.

\section{Wave physics}

In the presentation by Decker, quasilinear effect on ECRH was investigated in TCV and KSTAR. General agreement was obtained in $\mathrm{O} 2$ damping between diamagnetic loop measurements (DML) and LUKE simulations in TCV. No significant X2-O2 synergy was found either on DML or LUKE simulations. Ohkawa effect on ECCD was studied in KSTAR. No CD effect was observed for $\mathrm{Bt}=3.5 \mathrm{~T}$. So far off-axis ECCD, lower $\mathrm{T}_{\mathrm{e}}$ and $\mathrm{n}_{\mathrm{e}}$ yield the optical depth below unity. A significant part of the EC power crosses the resonance, which cancels out current drive effects.

Bae also studied synergetic effect of X2 and X3 EC waves in KSTAR. The $3 \mathrm{D}$ relativistic ray tracing and Fokker-Planck codes C3PO/LUKE were used for the quantitative prediction of its synergetic effect. First LUKE calculation shows nearly linear dependence of X3 absorption rate on X2 EC power and dependence of X3 absorption rate on $\mathrm{X} 2 \mathrm{EC}$ wave injection angle.

Takahashi presented direct measurement of power and refracted trajectory of transmitting EC beam through plasma on LHD. A target plate facing on the ECRH antenna was set in the vacuum vessel. In the $77 \mathrm{GHz}$ Omode experiments, a clear dependence of the beam refraction on the electron density gradient was observed from the landing-point of the EC beam on the target plate. The displacement of the EC beam trajectory due to the refraction well agreed with the ray trace calculation in the toroidal direction but not in the radial direction.

Kubo presented numerical analysis of efficient ECRH mode excitation through inhomogeneous peripheral plasma in LHD. When the scale lengths of plasma density, magnetic field strength and magnetic shear are comparable with the local wavelength, the coupling between X-and O-modes may be significant. A 1-D full wave calculation code was developed for the LHD configurations which solve electromagnetic wave equation, and incorporated in the ray tracing code, LHDGauss. 